\section{Avaliação do programa de promoção da atividade física Academia da Cidade de Recife, Pernambuco, Brasil: percepções de usuários e não-usuários}

\author{
Evaluation of the Academia da Cidade program to \\ promote physical activity in Recife, Pernambuco \\ State, Brazil: perceptions of users and non-users
}

\footnotetext{
${ }^{1}$ Departamento de Ginástica e Saúde, Universidade Federal de Pelotas, Pelotas, Brasil.

2 Departamento de Saúde, Faculdade dos Guararapes, Jaboatão dos Guararapes, Brasil.

3 Programa de Pós-graduação em Nutrição, Universidade Federal de Pernambuco, Recife, Brasil.

4 Programa de Pós-graduação em Educação Física,

Universidade Federal do Paraná, Curitiba, Brasil. ${ }^{5}$ Departamento de Pedagogia do Movimento do Corpo Humano, Universidade de São Paulo, São Paulo, Brasil.

${ }^{6}$ Coordenadoria de Doenças e Agravos Não-transmissíveis, Ministério da Saúde, Brasília, Brasil.

7 Programa Academia da Cidade, Secretaria de Saúde do Recife, Recife, Brasil.

Correspondência P. C. Hallal

Departamento de Ginástica e Saúde, Universidade Federal de Pelotas.

Rua Marechal Deodoro 1160

Pelotas, RS

96020-220, Brasil.

prchallal@terra.com.br
}

\author{
Pedro C. Hallal 1 \\ Maria Cecília Marinho Tenório 2 \\ Rafael Miranda Tassitano ${ }^{3}$ \\ Rodrigo Siqueira Reis 4 \\ Yara Maria Carvalho 5 \\ Danielle Keylla Alencar Cruz 6 \\ Wilson Damascena 7 \\ Deborah Carvalho Malta 6
}

\begin{abstract}
The aim of this study was to describe the profile of users and non-users of the Academia da Cidade Program to promote physical activity in Recife, Pernambuco State, Brazil. Four of the program's 19 units were sampled. Users ( $n=277)$ and nonusers ( $n=277)$ were matched for gender, age $( \pm 5$ years), and place of residence. Among non-users, $22 \%$ had never heard of the program. Among those who knew of the program, 54.3\% learned about it by actually having seen one of the units. Among users, the main reason for participating in the program was to improve their health; mean participation time was 27.5 months ( $S D$ = 23.1); and $72.8 \%$ reported high satisfaction. The proportion of individuals with fair or poor self-rated health was significantly higher among non-users (45.4\%) than users (28.5\%). Users of the program appeared to be satisfied. Strategies to further publicize the program should be prioritized to make it known to a larger proportion of the city's population.
\end{abstract}

Motor Activity; Health Programs and Plans; Health Promotion

\section{Introdução}

Nas últimas décadas, com o processo de industrialização, urbanização e mecanização, houve significativas mudanças no padrão de morbimortalidade mundial. No Brasil, em 2003, as doenças infectocontagiosas correspondiam a $5 \%$ do total do total de óbitos registrados, enquanto as doenças cardiovasculares e neoplasias correspondiam a $31 \%$ e $15 \%$, respectivamente 1 .

A falta de atividades físicas regulares vem sendo considerada um fator de risco primário e independente para muitos agravos à saúde, particularmente as doenças cardiovasculares e metabólicas 2,3,4. Apesar das evidências acumuladas sobre os benefícios da atividade física regular para a saúde, estudos têm indicado baixos níveis desse comportamento na população 5,6,7,8,9. Considerando apenas a atividade física no lazer, recente documento indica que, entre os adultos das capitais brasileiras, a prática de atividade física no lazer varia de 11,3\% em São Paulo a 20,5\% em Vitória (Espírito Santo) 10.

Esse quadro desfavorável no que se refere à prática de atividades físicas pela população motivou organizações internacionais e nacionais a incluírem a atividade física na agenda mundial de saúde pública. Com base na proposta das $\mathrm{Ci}$ dades Saudáveis, surgida em 1978 em Toronto, Canadá, algumas cidades brasileiras tiveram a iniciativa de desenvolver programas de promoção de atividade física, enfocando o aumento do 
nível de atividade física da população e a ampliação do conhecimento sobre os benefícios da sua prática 11. No Brasil, a recém-aprovada Política Nacional de Promoção da Saúde (PNPS) inclui a promoção de atividades físicas na agenda nacional. A Portaria $n^{\circ}$. 2.608, de 28 de dezembro de 2005 destina recursos a todos os estados da federação para investimento em projetos locais de incentivo à atividade física 12 .

Neste contexto, tem se observado um crescimento das iniciativas de larga abrangência populacional, sob a forma de programas e campanhas em prol de estilos de vida mais ativos ${ }^{13}$. No Recife (Pernambuco), o Programa Academia da Cidade foi implantado em 2002 pela Secretaria de Saúde do Recife e está hoje inserido no Sistema Único de Saúde (SUS) como uma política de atenção básica à saúde. O objetivo principal do programa é a promoção de atividades físicas, lazer e alimentação saudável para a comunidade. No entanto, existem poucos dados no que se refere ao impacto e relevância dessas iniciativas na comunidade e, conseqüentemente, da adesão da população aos programas. De fato, a participação nesses programas passa pela própria compreensão da população sobre a atividade física, saúde, corpo e qualidade de vida, além de outros conceitos 14; assim como pelos princípios que justificam tais iniciativas, tanto do poder público quanto do privado 15 .

A avaliação e problematização de tais questões não são freqüentes no campo da saúde, especialmente em programas de promoção de atividade física na comunidade, em parte pela falta de informações sobre os participantes dos programas. Nesse sentido, o presente artigo pretende descrever o perfil dos usuários e não-usuários do Programa Academia da Cidade em Recife.

\section{Métodos}

O presente estudo transversal faz parte da avaliação de programas de promoção de atividade física no Brasil, coordenada pelo Ministério da Saúde, Universidade Federal de Pelotas (UFPEL) e Universidade de São Paulo (USP). Este estudo foi submetido ao Comitê de Ética em Pesquisa com Seres Humanos da Universidade Federal de Pelotas e aprovado, acompanhando as normas estabelecidas nas Resoluções $n^{o}$. 196/96 e $n^{o}$. 251, do Conselho Nacional de Saúde.

O Programa Academia da Cidade é um programa público de promoção da saúde vinculado à Secretaria de Saúde do Recife. As atividades ocorrem durante a semana (segunda a sexta) em dois turnos (manhã e noite) em praças, parques e avenidas da cidade. Na época da coleta de dados deste estudo, existiam 19 pólos distribuídos pelos bairros de Recife. Para o estudo, optou-se por selecionar quatro pólos, sendo dois localizados em áreas ricas da cidade e dois localizados em áreas pobres. A seleção dos bairros foi baseada nos dados cadastrais do Programa Academia da Cidade. Além disso, recorremos ao Instituto Brasileiro de Geografia e Estatística (IBGE. Censo Demográfico de 2000. http://www.ibge.gov.br) para saber a renda familiar média dos chefes de família dos setores censitários que compõem os pólos sorteados. Para cada um dos bairros foram selecionados dois pólos, um com grande número de usuários e outro com baixo número de usuários.

As informações relacionadas ao bairro e à freqüência de usuários do programa foram obtidas por meio da coordenação do programa. Após o pareamento, foi realizado um sorteio para a seleção dos pares. Sendo assim, foi selecionado: um pólo rico e grande; um pólo rico e pequeno; um pólo pobre e grande e um pólo pobre e pequeno. Os pólos selecionados foram: Parque da Jaqueira, Lagoa do Araçá, Sítio da Trindade e Avenida do Forte.

A população de usuários do estudo foi constituída de todos os usuários dos quatro pólos selecionados. Adicionalmente, para cada usuário (caso), foi entrevistado um não-usuário (controle). Os não-usuários foram pareados aos casos em termos de vizinhança, sexo e idade ( \pm 5 anos). A coleta dos dados ocorreu durante o mês de agosto de 2007 e foi realizada por dois profissionais de educação física com experiência em coleta de dados em estudos epidemiológicos. O planejamento das visitas foi organizado de forma que cada pólo fosse visitado seis vezes, em dias não seqüenciais.

A coleta dos dados foi realizada por dois profissionais independentes do programa e que realizaram um treinamento prévio. As entrevistas com os usuários foram realizadas individualmente no respectivo pólo e tiveram duração média de dez minutos. Após todas as visitas previstas, iniciou-se a coleta das informações dos não-usuários. Mediante os dados cadastrais dos usuários, os avaliadores se direcionavam ao endereço do usuário e entrevistavam o vizinho do domicílio esquerdo. Caso o vizinho não contemplasse os critérios pré-estabelecidos, seguia-se até que fosse localizado um não-usuário que atendesse os critérios de inclusão.

As informações foram coletadas por meio de dois questionários (usuários e não-usuários), previamente testados. O questionário dos usuários continha informações sobre: conhecimento, participação e grau de satisfação com o programa, percepção de segurança no pólo e percepção 
de saúde, além de informações sócio-demográficas. Já o questionário dos não-usuários continha informações sobre: conhecimento e informação a respeito do programa, barreiras percebidas para a prática de atividade física, percepção de saúde, além de informações sócio-demográficas.

O procedimento de tabulação final dos dados foi efetuado no programa Epi Info (Centers for Disease Control and Prevention, Atlanta, Estados Unidos) e os dados foram analisados no programa Stata (Stata Corp., College Station, Estados Unidos). A análise dos dados incluiu procedimentos descritivos de estatística descritiva, e o teste do qui-quadrado para comparar a autopercepção de saúde entre usuários e não-usuários do programa.

\section{Resultados}

A amostra foi composta por 277 usuários e 277 não-usuários, totalizando 554 indivíduos (89,2\% de mulheres). Entre os usuários, 18 pessoas recusaram participar da pesquisa, enquanto entre os não-usuários a quantidade foi de 11. A idade média dos usuários do programa foi de 50 anos (desvio-padrão - DP = 14,4) e dos não-usuários de 49,1 anos (DP = 15,8), não havendo diferença significativa. Verificou-se uma maior quantidade de usuários nos pólos do Parque da Jaqueira ( $\mathrm{n}=75)$, Sítio da Trindade $(\mathrm{n}=89)$ e Lagoa do Araçá ( $\mathrm{n}=71$ ), em comparação ao pólo Avenida do Forte $(n=42)$. Na Tabela 1, encontra-se a distribuição da amostra total segundo o sexo e a faixa etária.
Entre os não-usuários, 22,2\% referiram nunca ter ouvido falar do programa. Entre os que conhecem o programa, $54,3 \%$ o conhecem por terem visto um pólo, enquanto $21,6 \%$ souberam do programa por meio de outra pessoa. Entre os que já participaram do programa, 90,6\% deixaram de participar por motivos pessoais e $9,4 \%$ por motivos relacionados ao programa. Entre os motivos pessoais, destacam-se a falta de tempo $(37,5 \%)$ doença (15,6\%), distância do pólo (15,6\%), prática de atividade física em outro local $(9,4 \%)$, falta de coragem pessoal em freqüentar um programa de atividade física $(9,4 \%)$ e gestação $(3,1 \%)$. O horário das aulas $(6,3 \%)$, a percepção de que o programa era fraco e a falta de material $(3,1 \%)$ foram os outros motivos relatados para a desistência do programa.

Quando perguntados sobre o que seria necessário para freqüentarem o programa, os nãousuários relataram, sobretudo: ter mais tempo $(32,2 \%)$, ter vontade, disposição ou um incentivo (16,8\%), criação de pólos mais próximos de suas residências $(6,9 \%)$. Ainda foi verificado que 3,5\% relataram que seria necessária mudança nas aulas e/ou professores e $2 \%$ um melhoramento ambiental do pólo. Apenas 5,4\% relataram não ter interesse em participar.

Entre os usuários a maioria relatou que ficou sabendo do programa por ter visto um pólo (65\%) ou por meio de outra pessoa (28,9\%). Quando perguntados sobre a principal razão para a participação no programa, 65,7\% responderam por saúde, enquanto $17,3 \%$ por recomendação médica. Outros motivos relatados foram: motivos estéticos $(6,9 \%)$, preencher o tempo livre $(3,2 \%)$,

Tabela 1

Distribuição sócio-demográfica dos usuários e não-usuários do Programa Academia da Cidade, segundo sexo e faixa etária.

\begin{tabular}{|c|c|c|c|c|c|c|c|c|}
\hline \multirow[t]{3}{*}{ Variável } & \multicolumn{2}{|c|}{ Parque da Jaqueira } & \multicolumn{2}{|c|}{ Lagoa do Araçá } & \multicolumn{2}{|c|}{ Sítio da Trindade } & \multicolumn{2}{|c|}{ Avenida do Forte } \\
\hline & Caso & Controle & Caso & Controle & Caso & Controle & Caso & Controle \\
\hline & $\%(n)$ & $\%(n)$ & $\%(n)$ & $\%(n)$ & $\%(n)$ & $\%(n)$ & $\%(n)$ & $\%(n)$ \\
\hline \multicolumn{9}{|l|}{ Sexo } \\
\hline Masculino & $14,7(11)$ & $14,7(11)$ & $4,2(3)$ & $4,2(3)$ & $14,6(13)$ & $14,6(13)$ & $7,1(3)$ & $7,1(3)$ \\
\hline Feminino & $85,3(64)$ & $85,3(64)$ & $95,8(68)$ & $95,8(68)$ & $85,4(76)$ & $85,4(76)$ & $92,9(39)$ & 92,9 (39) \\
\hline Valor de $p^{*}$ & \multicolumn{2}{|c|}{1,0} & \multicolumn{2}{|c|}{1,0} & \multicolumn{2}{|c|}{1,0} & \multicolumn{2}{|c|}{1,0} \\
\hline \multicolumn{9}{|l|}{ Idade (anos) } \\
\hline $16-30$ & $13,3(10)$ & $7,3(13)$ & $12,2(9)$ & $15,5(11)$ & $1,2(10)$ & $15,7(14)$ & $2,4(1)$ & $2,4(1)$ \\
\hline $31-40$ & $6,7(5)$ & $9,3(7)$ & $11,2(8)$ & $21,1(15)$ & $28,1(25)$ & 21,5 (19) & $14,3(6)$ & $9,5(4)$ \\
\hline $41-50$ & $14,7(11)$ & $14,6(11)$ & $19,7(14)$ & $16,9(12)$ & $23,6(21)$ & $26,9(24)$ & $16,7(7)$ & $31,0(13)$ \\
\hline $51-60$ & $30,7(23)$ & $26,6(20)$ & $29,5(21)$ & $21,1(15)$ & $21,3(19)$ & $20,2(18)$ & $42,9(18)$ & $35,7(15)$ \\
\hline 60 ou mais & $34,7(26)$ & $32,0(24)$ & $26,7(19)$ & $25,4(18)$ & $15,7(14)$ & $15,7(14)$ & $23,8(10)$ & $21,4(9)$ \\
\hline Valor de $p^{*}$ & \multicolumn{2}{|c|}{0,92} & \multicolumn{2}{|c|}{0,84} & \multicolumn{2}{|c|}{0,92} & \multicolumn{2}{|c|}{0,89} \\
\hline
\end{tabular}

* Teste do qui-quadrado. 
aumentar a rede social $(3,2 \%)$, lazer $(0,7 \%)$, alto custo de uma academia particular $(1,1 \%)$, melhorar a auto-estima $(0,4 \%)$, orientação para atividade física $(1,1 \%)$ e recomendação nutricional $(0,4 \%)$.

O tempo médio de participação dos usuários no programa foi de 27,5 meses (DP $=23,1)$. Quando estratificado entre os pólos, essa média foi de 33,0 (DP = 27,4) no pólo do Parque da Jaqueira; 23,2 (DP = 22,8) no Sítio da Trindade; 33,0 $(\mathrm{DP}=33,1)$ na Avenida do Forte e; 24,1 (DP = 15,7) na Lagoa do Araçá. Em relação à participação no programa, a maioria o freqüenta quatro vezes ou mais por semana. Quando avaliado o tempo diário que os usuários praticam as atividades no programa, a média encontrada foi de 69 minutos ( $\mathrm{DP}=22,3$ ). Na Tabela 2, encontramse informações sobre a participação dos usuários do programa, estratificadas por pólo.

Quando questionados com a pergunta "Você se considera participante da construção das atividades desenvolvidas no pólo?", um pouco mais da metade dos usuários (56,7\%) relatou que não. No Parque da Jaqueira, o percentual foi de $42,7 \%$; no Sítio da trindade 59,6\%; na Avenida do Forte $50 \%$; e na Lagoa do Araçá $71,8 \%$. Seis em cada dez usuários relataram encontrar pessoas que já conheciam antes de decidir participar das atividades.

O tempo médio gasto pelos usuários para se deslocar da sua residência para o pólo foi de 15,2 minutos (DP $\pm 10,0$ ). Quando estratificado entre os pólos, observou-se que no pólo do Parque da Jaqueira a média foi de 16,2 minutos (DP $\pm 12,0$ ), no Sítio da Trindade 19,5 minutos (DP $\pm 9,9$ ), na Avenida do Forte 12,6 minutos (DP $\pm 6,8$ ) e na Lagoa do Araçá 10,2 minutos (DP $\pm 6,2$ ). A maioria relatou ir para o pólo a pé $(79,4 \%)$. Outras formas de deslocamento para o pólo foram citadas como: ônibus (9\%), carro $(6,9 \%)$ e bicicleta $(3,6 \%)$. A Tabela 3 apresenta a forma e o tempo de deslocamento, estratificada por pólo.

Na avaliação dos usuários, apenas 1,1\% relatou que o programa não atinge os objetivos, enquanto $64,3 \%$ acreditam que atingem totalmente e $34,7 \%$ que atinge parcialmente. Não foi observada diferença significativa entre os pólos. Quando avaliado o grau de satisfação com o programa, $72,8 \%$ dos usuários relataram um grau elevado (alto ou muito alto) e $23,2 \%$ grau de satisfação médio e apenas $4 \%$ consideram baixo ou insatisfeito. A Figura 1 apresenta o grau de satisfação dos usuários do programa, estratificado por pólo.

Quando perguntados "Se você pudesse modificar algo no programa, o que seria?”, $61 \%$ dos usuários responderam que melhorariam a estrutura, incluindo a aquisição de mais e novos materiais, enquanto $11,6 \%$ não modificariam nada. Outras mudanças sugeridas foram: contratação de mais profissionais (2,3\%), maior divulgação $(6,5 \%)$, qualidade das aulas $(8,7 \%)$, ampliação do serviço $(0,4 \%)$, avaliação física mais constante $(1,2 \%)$, mais profissionais de educação física

Tabela 2

Participação e freqüência semanal dos usuários do Programa Academia da Cidade, estratificado por pólo.

\begin{tabular}{|c|c|c|c|c|}
\hline \multirow[t]{2}{*}{ Variáveis } & Parque da Jaqueira & Lagoa do Araçá & Sítio da Trindade & Avenida do Forte \\
\hline & $\%(n)$ & $\%(n)$ & $\%(n)$ & $\%(n)$ \\
\hline \multicolumn{5}{|c|}{ Tempo de participação no programa (meses) } \\
\hline $1-6$ & $28,0(21)$ & $23,9(17)$ & 43,8 (39) & $26,2(11)$ \\
\hline $7-12$ & $13,3(10)$ & $12,7(9)$ & $6,7(6)$ & $4,8(2)$ \\
\hline 13 ou mais & $58,7(44)$ & $63,4(45)$ & $49,4(44)$ & $69,0(29)$ \\
\hline \multicolumn{5}{|l|}{ Freqüência semanal } \\
\hline Até 3 vezes & $32,4(23)$ & $16,9(12)$ & $20,9(18)$ & $14,3(6)$ \\
\hline $4-5$ vezes & $67,6(48)$ & $83,1(59)$ & $79,1(68)$ & $85,7(36)$ \\
\hline \multicolumn{5}{|c|}{ Tempo diário de permanência no programa (minutos) } \\
\hline Até 60 & $77,3(68)$ & $93,0(66)$ & $64,0(57)$ & $73,8(31)$ \\
\hline $61-90$ & $12,0(9)$ & $4,2(3)$ & $22,5(20)$ & $16,7(7)$ \\
\hline Acima de 90 & $10,7(8)$ & $2,8(2)$ & $13,5(12)$ & $9,5(5)$ \\
\hline \multicolumn{5}{|l|}{ Atividades realizadas } \\
\hline Ginástica & $78,7(59)$ & $80,3(57)$ & $36,0(32)$ & $59,5(25)$ \\
\hline Caminhada orientada & $1,3(1)$ & $0,0(0)$ & $3,4(3)$ & $2,4(1)$ \\
\hline Ginástica + caminhada orientada & $20,0(15)$ & $19,7(14)$ & $60,7(54)$ & $38,1(16)$ \\
\hline
\end{tabular}


Tabela 3

Modo de deslocamento para o pólo e tempo gasto (estratificado por pólo) entre os usuários do Programa Academia da Cidade.

\begin{tabular}{|c|c|c|c|c|}
\hline \multirow[t]{2}{*}{ Variáveis } & Parque da Jaqueira & Lagoa do Araçá & Sítio da Trindade & \multirow{2}{*}{$\begin{array}{l}\text { Avenida do Forte } \\
\qquad \%(n)\end{array}$} \\
\hline & $\%(n)$ & $\%(n)$ & $\%(n)$ & \\
\hline \multicolumn{5}{|c|}{ Meio de transporte } \\
\hline Ativo & $60,0(45)$ & $91,5(65)$ & $92,1(82)$ & $97,6(41)$ \\
\hline Passivo & $40,0(30)$ & $13,6(6)$ & $7,9(7)$ & $2,3(1)$ \\
\hline \multicolumn{5}{|c|}{ Distância (minutos) } \\
\hline Até 15 & $65,3(49)$ & $87,3(62)$ & $52,8(47)$ & $76,2(32)$ \\
\hline $16-30$ & $28,0(21)$ & 12,7 (9) & $39,3(35)$ & $23,8(10)$ \\
\hline 31 ou mais & $6,7(5)$ & $0,0(0)$ & $7,9(7)$ & $0,0(0)$ \\
\hline
\end{tabular}

$(1,2 \%)$, bebedouro $(0,4 \%)$, médicos e nutricionistas $(1,2 \%)$, segurança $(0,4 \%)$ e fardamento $(0,8 \%)$. Quando perguntados “Em sua opinião, quais as perspectivas para o Programa Academia da Cidade?", apenas 5,5\% acreditam que o programa tende a acabar, enquanto $39,3 \%$ acreditam que tende a manter-se como está e 55,3\% acreditam que o programa crescerá.

O percentual de pessoas com percepção excelente ou muito boa de saúde foi similar entre usuários e não-usuários do programa (Figura 2). Já o percentual de indivíduos com percepção de saúde regular ou ruim foi significativamente maior entre os não-usuários $(45,4 \%)$ do programa, em comparação aos usuários (28,5\%).

A percepção elevada de segurança foi significativamente maior entre os usuários do pólo do Parque da Jaqueira $(64,9 \%)$ em comparação aos demais pólos. Nos pólos do Sítio da Trindade e Avenida do Forte predominou uma percepção média (57,3\% e 40,5\%, respectivamente), enquanto na Lagoa do Araçá se encontrou uma percepção predominante de baixa segurança $(42,9 \%)$ (Figura 3 ).

\section{Discussão}

Este estudo, de delineamento transversal, avaliou o Programa Academia da Cidade, em Recife, descrevendo o perfil dos usuários e não-usuários. Ainda que se tenha verificado aumento no número de intervenções em promoção da atividade física na América Latina, a escassez da literatura contendo descrições das avaliações de intervenções em atividade física em nível comunitário foi uma constatação de uma recente revisão sistemática 16. Ainda se observou a necessidade de publicação e divulgação dos resultados oriundos de intervenções avaliadas, para que se crie um conjunto de evidências sobre a efetividade dos programas 16 .

Estudo recentemente publicado teve como objetivo avaliar o impacto do programa em relação ao nível de atividade física da população com mais de 16 anos da cidade do Recife ${ }^{17}$. As entrevistas foram realizadas por telefone $(n=2.047)$ a respeito da prática de atividade física moderada a vigorosa e a participação no programa. Os resultados indicam que aquelas pessoas que participaram do programa no passado (odds ratio - OR = 2,0;intervalo de 95\% de confiança - IC95\%: $1,0-3,9)$ e que participam no presente $(\mathrm{OR}=11,3$; IC95\%: 3,5-35,9) apresentam maior chance de serem fisicamente ativas no lazer em comparação àquelas que nunca participaram. Entretanto apenas 5,2\% dos entrevistados relataram ter participado ou estarem participando das atividades do programa 17.

A literatura revela que a adesão à prática de atividades físicas sofre influência de fatores, como: experiências anteriores na prática esportiva e de exercícios físicos; apoio do cônjuge e de familiares 18; aconselhamento médico 19; conveniência do local de exercitação 20; gênero 21; disponibilidade de tempo 22; condição sócio-econômica; conhecimento sobre exercício físico e acesso a instalações e espaços adequados à prática de exercícios físicos 23,24 . No presente estudo, os fatores de ordem pessoal (falta de tempo, doença e vontade) foram os mais determinantes para a desistência das atividades oferecidas, independente do pólo.

Os estudos sobre a influência do ambiente físico na adesão ao exercício demonstram que o acesso a instalações é uma variável ambiental que interfere na escolha da pessoa em se exercitar 25. A riqueza na oferta de instalações para a 
Figura 1

Grau de satisfação dos usuários com o Programa Academia da Cidade, estratificado por pólo.

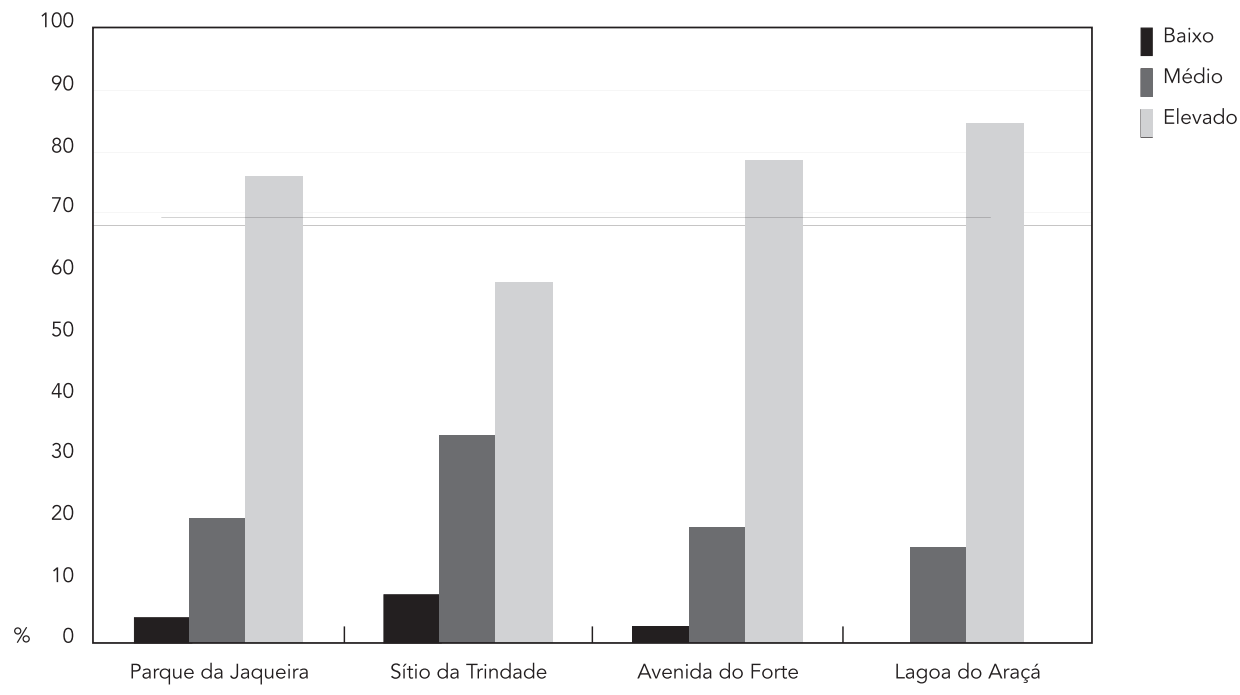

Figura 2

Percepção de saúde dos usuários e não-usuários do Programa Academia da Cidade.

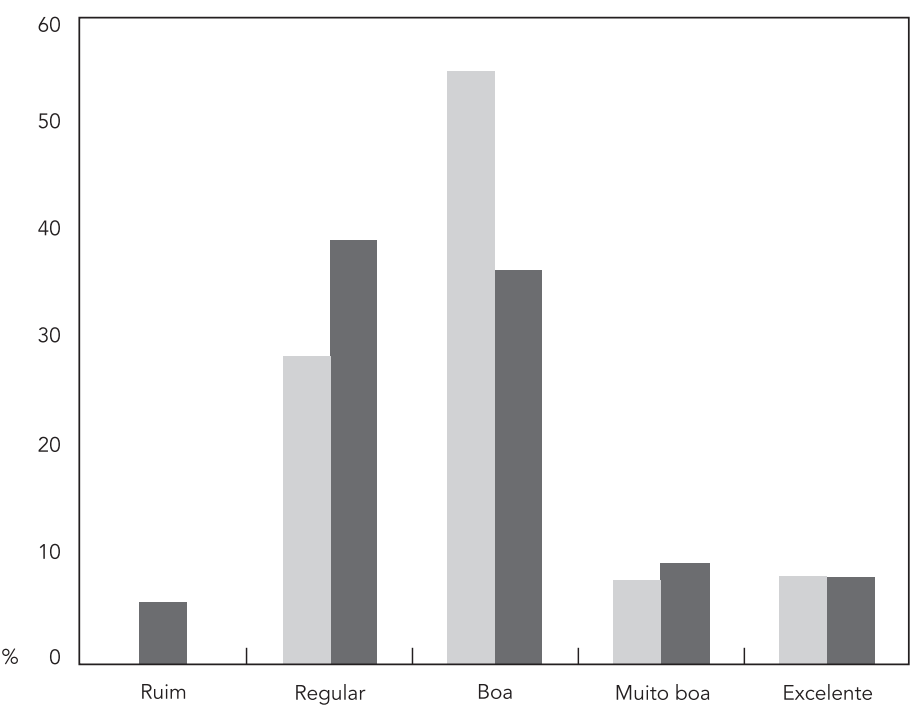




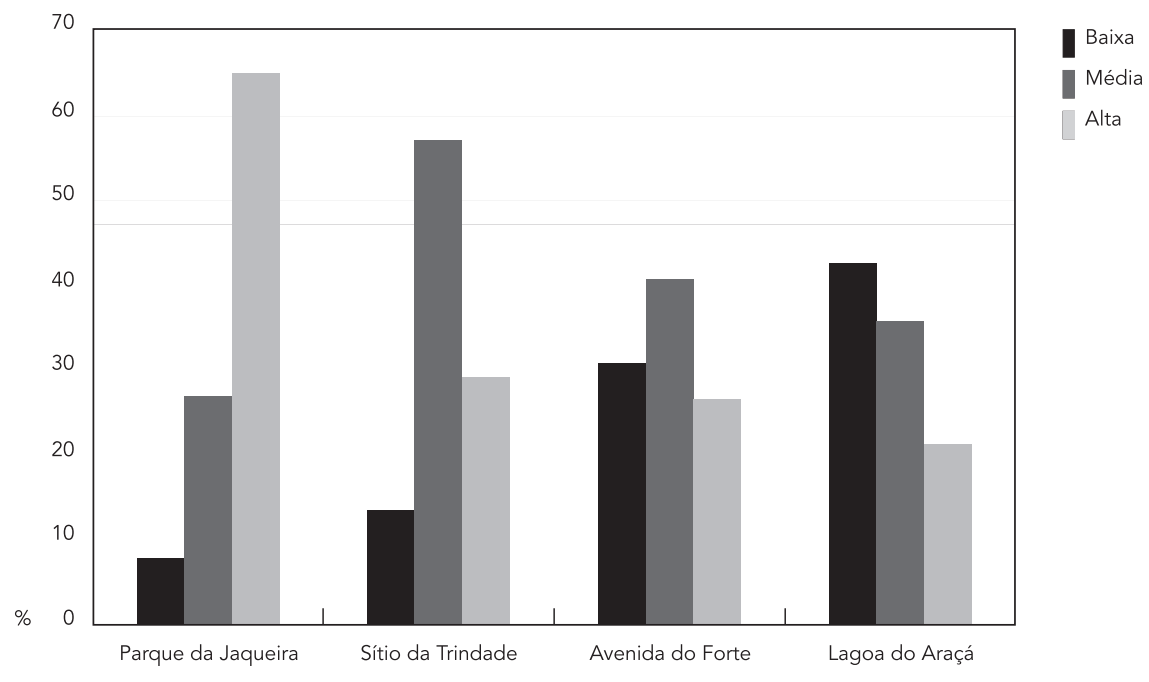

prática do exercício, além de servir de estímulo visual, chamando a atenção das pessoas para a questão da prática do exercício, reduz barreiras físicas e psicológicas associadas ao exercício, uma vez que a proximidade do local para a prática diminui o tempo de deslocamento da pessoa 26. Os pólos do programa são localizados em praças e parques da cidade, o que facilita o acesso potencial as atividades, porque são locais públicos. Todavia, a grande distância da residência até os pólos, bem como a percepção de pouca segurança, podem constituir uma barreira na adesão do programa.

Estudo realizado nas 27 capitais do Brasil mostrou que $14,9 \%$ dos habitantes são ativas no lazer, e tal prática predomina entre homens (18,3\%) e jovens de 18 a 24 anos (18,3\%). O mesmo perfil se repete na cidade do Recife 27 . O Programa Academia da Cidade tem criado oportunidade de atividade física no lazer para outros estratos populacionais que habitualmente não têm muitas oportunidades na prática de atividade física no lazer, como as mulheres, que constituem $89,2 \%$ das usuárias do programa e em idade superior à média nacional (Censo Demográfico de 2000. http://www.ibge.gov.br): 50 anos (DP = $14,4)$. Outro resultado a ser destacado consiste na mudança dos usuários também em relação à adoção de novos hábitos alimentares (48,2\%), mostrando a importância na associação da recomendação da Organização Mundial da Saúde
(OMS) da estratégia global de atividade física e alimentação saudável ${ }^{28}$. A baixa freqüência de participação dos homens no programa pode estar relacionada à visualização das atividades oferecidas nos pólos. Apesar da oferta de caminhadas orientadas, as aulas de ginástica, atividade de preferência das mulheres, ocorrem em espaços abertos e com músicas, sendo mais visíveis para a população em geral.

O Programa Academia da Cidade promove a difusão da atividade física para a população em geral por meio da sua identidade visual. Os usuários chegaram ao programa na sua maioria (65\%) por terem visto o programa, e entre nãousuários, $(54,3 \%)$ foram informados do programa por terem visto suas instalações. Esse fato pode ser explicado pela distribuição do programa nas praças, parques, praias da cidade, ao longo das avenidas, fazendo parte de reformulação do espaço urbano. Outra forma de transmissão do programa consiste na transmissão oral, tanto entre usuários $(28,9 \%)$, quanto entre não-usuários $(21,6 \%)$. Estes dados também foram registrados por Simões et al. 17, no inquérito populacional realizado em 2007 em Recife.

Nesse contexto, intervenções ambientais e na área de políticas públicas são particularmente importantes para a promoção da atividade física, porque ambas são elaboradas para influenciar grandes grupos populacionais por meio da oferta de espaços e serviços públicos. Além da quanti- 
dade de pessoas que podem se beneficiar de tais iniciativas, aumentando assim o nível de atividade física populacional, outras características devem ser consideradas. Aspectos relacionados à oferta, acessibilidade e qualidade de espaços devem ser mais bem abordados para traduzir de forma mais correta o desempenho das ações adotadas, tornando mais consistentes a elaboração e a avaliação desses programas de promoção de atividade física na comunidade.

De uma maneira geral, os entrevistados que participam do programa estão satisfeitos com as atividades oferecidas e com os profissionais do programa. Tanto entre os que nunca participaram ou os que já participaram no passado, as principais barreiras são de ordem pessoal. Atualmente, o Programa Academia da Cidade está expandindo, com a criação de novos pólos na cidade de Recife e a ampliação do programa para outras cidades do Estado de Pernambuco e mesmo de outros estados. Ao longo dessa expansão, estratégias de divulgação devem ser priorizadas para que o programa seja conhecido por um maior percentual de residentes de Recife.

Avaliações periódicas devem ser realizadas para confirmar a efetividade do programa e para avaliar sua estrutura e processo.

\section{Resumo}

O objetivo foi descrever usuários e não-usuários do Programa Academia da Cidade de Recife, Pernambuco, Brasil. Foram selecionados quatro dos 19 pólos do programa. Para cada usuário sorteado, foi selecionado um não-usuário, que foi pareado ao usuário em termos de sexo, idade ( \pm 5 anos) e local de residência. A amostra foi composta por 277 usuários e 277 nãousuários. Entre os não-usuários, $22 \%$ referiram nunca ter ouvido falar do programa. Entre os que já ouviram falar do programa, 54,3\% o conhecem por terem visto um pólo. Entre os usuários, o principal motivo para a participação no programa foi "melhorar a saúde"; tempo médio de participação no programa foi de 27,5 meses $(D P=23,1)$ e $72,8 \%$ relataram grau elevado de satisfação. O percentual de pessoas com percepção de saúde regular ou ruim foi significativamente maior entre os não-usuários $(45,4 \%)$ em comparação aos usuários (28,5\%). Os usuários do Programa Academia da Cidade parecem estar satisfeitos. Estratégias de divulgação devem ser priorizadas para que o programa seja conhecido por um maior percentual de residentes de Recife.

Atividade Motora; Planos e Programas de Saúde; Promoção da Saúde

\section{Colaboradores}

P. C. Hallal coordena a rede de avaliação de programas de atividade física do Brasil, liderou a escrita do artigo e as análises dos dados. M. C. M. Tenório e R. M. Tassitano coordenaram a coleta de dados e participaram da análise de dados e da redação do artigo. R. S. Reis, D. K. A. Cruz e D. C. Malta coordenam a rede de avaliação de programas de atividade física do Brasil e colaboraram na redação do artigo. Y. M. Carvalho coordenou o estudo qualitativo com usuários, não-usuários e professores do Programa Academia da Cidade e aprovou a versão final do manuscrito. W. Damascena coordena o Programa Academia da Cidade, participou da elaboração do instrumento de pesquisa e aprovou a versão final do manuscrito. 


\section{Referências}

1. Ministério da Saúde. A vigilância, o controle e a prevenção das doenças não-transmissíveis: DCNT no contexto do Sistema único de Saúde Brasileiro. Brasília: Ministério da Saúde/Organização PanAmericana da Saúde; 2005.

2. Török K, Szelényi Z, Pórszász J, Molnár D. Low physical performance in obese adolescent boys with metabolic syndrome. Int J Obes Relat Metab Disord 2001; 25:966-70.

3. Ciolac EG, Guimarães GV. Exercício físico e síndrome metabólica. Rev Bras Med Esporte 2004; 10:319-24

4. Katzmarzyk PT, Church TS, Janssen I, Ross R, Blair SN. Metabolic syndrome, obesity, and mortality: impact of cardiorespiratory fitness. Diabetes Care 2005; 28:391-7.

5. Barros MVG, Nahas MV. Comportamentos de risco, auto-avaliação do nível de saúde e percepção de estresse entre trabalhadores da indústria. Rev Saúde Pública 2001; 35:554-63.

6. Masson CR, Dias-da-Costa JS, Olinto MTA, Meneghel S, Costa CC; Bairros F, et al. Prevalência de sedentarismo nas mulheres adultas da Cidade de São Leopoldo, Rio Grande do Sul, Brasil. Cad Saúde Pública 2005; 21:1685-95.

7. Hallal PC, Matsudo SM, Matsudo VKR, Araújo TL, Andrade DR, Bertoldi AD. Physical activity in adults from two Brazilian areas: similarities and differences. Cad Saúde Pública 2005; 21:573-80.

8. Hallal PC, Victora CG, Wells JC, Lima RC. Physical inactivity: prevalence and associated variables in Brazilian adults. Med Sci Sports Exerc 2003; 35:1894-900.

9. Pitanga FJG, Lessa I. Prevalência e fatores associados ao sedentarismo no lazer em adultos. Cad Saúde Pública 2005; 21:870-77.

10. Ministério da Saúde. VIGITEL Brasil 2007: vigilância de fatores de risco e proteção para doenças crônicas por inquérito telefônico. Brasília: Ministério da Saúde; 2008.

11. Adriano J, Werneck G, Santos M, Souza R. A construção de cidades saudáveis: uma estratégia viável para a melhoria da qualidade de vida? Ciênc Saúde Coletiva 2000; 5:53-62.

12. Ministério da Saúde. Política Nacional de Promoção da Saúde. Brasília: Ministério da Saúde; 2006. (Série Pactos pela Saúde, 7).

13. Ferreira MS, Najar AL. Programas e campanhas de promoção da atividade física. Ciênc Saúde Coletiva 2005 ; 10 Suppl:207-19.

14. Carvalho YM. Atividade física e saúde: onde está e quem é o sujeito da relação? Rev Bras Ciênc Esporte $2001 ; 22: 9-21$.

15. Carvalho YM. Saúde, sociedade e vida: um olhar da Educação Física. Rev Bras Ciênc Esporte 2006; 27:153-68.
16. Hoehner CM, Soares J, Parra Perez D, Ribeiro IC, Joshu CE, Pratt M, et al. Physical activity interventions in Latin America: a systematic review. Am J Prev Med 2008; 34:224-33.

17. Simões EJ, Hallal P, Pratt M, Ramos L, Munk M, Damascena W, et al. Effects of community-based, professionally supervised intervention on physical activity levels among residents of Recife, Brazil. Am J Public Health 2009; 99:68-75.

18. King AC, Blair SN, Bild DE, Dishman RK, Dubbert PM, Marcus BH, et al. Determinants of physical activity and interventions in adults. Med Sci Sports Exerc 1992; 24:S221-36.

19. Bull FC, Jamrozik K. Advice on exercise from a family physician can help sedentary patients to become active. Am J Prev Med 1998; 15:85-94.

20. Andrew GM, Oldridge NB, Parker JO, Cunningham DA, Rechnitzer PA, Jones NL, et al. Reasons for dropout from exercise programmes in post-coronary patients. Med Sci Sports Exerc 1981; 13:164-8.

21. Garcia AW, Broda MA, Frenn M, Coviak C, Pender NJ, Ronis DL. Gender and developmental differences in exercise beliefs among youth and prediction of their exercise behavior. J Sch Health 1995; 65:213-9.

22 Johnson CA, Corrigan SA, Dubbert PM, Gramling SE. Perceived barriers to exercise and weight control practices in community women. Women Health 1990; 16:177-91.

23. Sallis JF, Prochaska JJ, Taylor WC. A review of correlates of physical activity of children and adolescents. Med Sci Sports Exerc 2000; 32:963-75.

24. Welk GJ. The youth physical activity promotion model: a conceptual bridge between theory and practice. Quest 1999; 51:5-23.

25. Sallis JF, Hovell MF, Hofstetter CR, Elder JP, Hackley M, Caspersen CJ, et al. Distance between homes and exercise facilities related to frequency of exercise among San Diego residents. Public Health Rep 1990; 105:179-85.

26. Hunter D. Community determinants and benefits of active living. In: Quinney HA, Gauvin L, Wall AET, editors. Toward active living: proceedings of the international conference on physical activity, fitness and health. Champaign: Human Kinetics; 1994. p. 203-6.

27. Malta DC, Castro AM, Allencar DK, Gosh CS. A Política Nacional de Promoção da Saúde e a agenda da Atividade física no contexto do SUS. Epidemiol Serv Saúde 2009; 18:7-16.

28. World Health Organization. WHO global strategy on diet, physical activity and health. Food Nutr Bull 2004; 25:292-302.

Recebido em 06/Mar/2009

Versão final reapresentada em 24/Set/2009 Aprovado em 21/Out/2009 